Revue des patrimoines

$37 \mid 2018$

Jardins collectifs : de l'abbé Lemire aux jardins d'insertion. Typologies - Expériences - Enjeux de conservation

\title{
Des jardins ouvriers au jardinage de rue : pour une géohistoire des jardins collectifs à Marseille
}

From workers' allotment gardens to street gardens: a geo-history of collective gardens in Marseilles

Jean Noël Consalès

\section{(2) OpenEdition}

Journals

Édition électronique

URL : http://journals.openedition.org/insitu/19252

DOI : $10.4000 /$ insitu. 19252

ISSN : 1630-7305

Éditeur

Ministère de la culture

Référence électronique

Jean Noël Consalès, « Des jardins ouvriers au jardinage de rue : pour une géohistoire des jardins collectifs à Marseille », In Situ [En ligne], 37 | 2018, mis en ligne le 12 décembre 2018, consulté le 19 avril 2019. URL : http://journals.openedition.org/insitu/19252 ; DOI : 10.4000/insitu.19252

Ce document a été généré automatiquement le 19 avril 2019

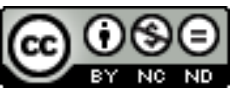

In Situ Revues des patrimoines est mis à disposition selon les termes de la licence Creative Commons Attribution - Pas d'Utilisation Commerciale - Pas de Modification 4.0 International. 


\title{
Des jardins ouvriers au jardinage de rue : pour une géohistoire des jardins collectifs à Marseille
}

\author{
From workers' allotment gardens to street gardens: a geo-history of collective \\ gardens in Marseilles
}

Jean Noël Consalès

\section{Introduction : l'espace et le temps des jardins collectifs à Marseille ${ }^{1}$}

1 Même si elle n'a pas abouti, la proposition de loi de $2003^{2}$ a consacré, dans l'usage courant, le terme de "jardins collectifs " pour qualifier de manière générique toutes les formes de potagers urbains cultivés par une communauté de jardiniers. Force est cependant de constater que derrière ce terme intégrateur se cachent des réalités territoriales très variées, désignées par des dénominations diverses et héritées de périodes historiques distinctes. Entre les jardins ouvriers issus de la fin du XIX $\mathrm{x}^{\mathrm{e}}$ siècle et les formes collectives de jardinage de rue développées depuis 2010 se trouvent ainsi plusieurs types de jardins collectifs qui renvoient non seulement à des logiques de création différentes mais encore à des logiques d'implantation particulières.

Notre objectif est donc ici de contribuer à retracer une géohistoire ${ }^{3}$ de ces jardins collectifs, en identifiant pour chaque période de création d'une forme spécifique de potagers urbains, les pensées qui ont engendré son émergence, les acteurs qui ont porté sa dynamique et les espaces urbains qui lui sont dédiés. En partant du contexte idéologique global, nous nous proposons d'étudier plus d'un siècle de jardins collectifs, à l'échelle locale, à partir du cas marseillais. Avec ses 12 sites de jardins familiaux, dont certains datent du début $d u x^{e}$ siècle, ses quelque 53 sites de jardins partagés et sa dynamique marquée de jardinage de rue, Marseille constitue en effet un cadre privilégié d'étude des potagers urbains sur le temps long. 
3 Deux parties distingueront les différentes formes de jardins collectifs associées aux périodes d'évolution qu'a permis de dégager la combinaison de plusieurs approches méthodologiques. Dans une première partie est exposé le processus territorial qui conduit les jardins ouvriers sis dans les faubourgs agricoles du début du $\mathrm{Xx}^{\mathrm{e}}$ siècle à devenir les jardins familiaux pris dans les tensions foncières des banlieues résidentielles actuelles. Pour ce faire, le propos s'appuie sur une étude historique des registres ${ }^{4}$ de l'Euvre des jardins de famille, ancêtre direct du comité local marseillais de la Fédération nationale des jardins familiaux et collectifs (FNJFC) $)^{5}$. Bien que centrés sur le cas d'une seule et même institution, ces documents permettent, à défaut d'établir un recensement exhaustif des groupes de potagers à travers le temps ${ }^{6}$, de dégager les logiques d'implantation précises des jardins ouvriers et familiaux au cours du $\mathrm{xx}^{\mathrm{e}}$ siècle. L'ensemble des sites investis par l'œuure des jardins de famille de 1910 à 1945 a en effet été identifié et précisément reporté sur les levés cartographiques au $1 / 10000^{\circ}$ réalisés par le Service géographique de l'armée au début $\mathrm{du} \mathrm{xx}^{\mathrm{e}}$ siècle (voir fig. 1). Offrant une grande précision sur les formes d'occupation du sol, ces relevés fournissent des informations essentielles quant à la répartition des groupes de potagers au sein de banlieues agricoles dont la physionomie n'a guère évolué jusqu'aux Trente Glorieuses. La figure 2 spatialise, pour sa part, un inventaire des jardins familiaux présents sur la commune de Marseille en 2017.

4 Dans une seconde partie, nous décrirons le processus de diversification des formes de jardins collectifs, à partir des observations réalisées au long cours selon deux postures de recherche, géographique et urbanistique, différentes :

5 - une posture détachée, dans le cadre d'une démarche scientifique initiée par un DEA, en 1998 ${ }^{7}$, poursuivie par une thèse de doctorat ${ }^{8}$, de 1999 à 2004, et ponctuée par la codirection du programme ANR JASSUR ${ }^{9}$;

6 - une posture impliquée, dans le cadre d'un engagement associatif et fédératif permettant de pratiquer, sur le terrain, des approches tantôt d'observation participante, tantôt de participation observante ${ }^{10}$.

7 Les figures 3 et 4 spatialisent les observations réalisées pour les jardins partagés et le jardinage de rue.

\section{Des jardins ouvriers dans les banlieues agricoles aux jardins familiaux dans les banlieues résidentielles}

Les jardins ouvriers sont apparus en France durant la révolution industrielle. Ils trouvent leur origine dans le mouvement terrianiste ${ }^{11}$ et doivent beaucoup à l'une de ses figures tutélaires, l'abbé Lemire. Élu plusieurs fois à l'Assemblée nationale et à la mairie de Hazebrouck, cet ecclésiastique crée en effet, en 1896, la Ligue française du coin de terre et du foyer (LFCTF), afin de promouvoir les potagers urbains. Les fonctions assignées aux jardins ouvriers sont, à l'époque, larges et diverses. Selon l'abbé Lemire, outre leurs valeurs moralisatrices et sociales (lutte contre l'alcoolisme, attachement à la terre et défense de la famille), ils permettent de réduire la misère ouvrière, grâce à l'amélioration de l'économie familiale. De fait, le mouvement social engagé par la LFCTF donne naissance à un véritable modèle de jardin nourricier se définissant, aujourd'hui, comme « les terrains divisés en parcelles, affectés par les collectivités territoriales ou par les associations à des particuliers y pratiquant le jardinage pour leurs propres besoins et 
ceux de leur famille, à l'exclusion de tout usage commercial [...] $»^{12}$. À son origine, ce modèle de jardins est marqué par ses logiques d'implantation particulières. Dans les régions industrielles, où les besoins ouvriers sont les plus nombreux, la grande majorité des sites de potagers s'insère à la périphérie des villes, au sein des nouveaux faubourgs industriels qui s'édifient, au-delà des anciens remparts, sur les terres cultivées des banlieues agricoles traditionnelles ${ }^{13}$. La figure 1 démontre ainsi qu'à Marseille, de 1910 à 1945, toutes les réalisations portées par la principale association de jardins ouvriers, l'Cuuve des jardins de famille, se font sur l'armature du vaste terradou ${ }^{14}$. C'est en effet au sein de ces faubourgs agricoles en cours d'industrialisation que se fixent les populations ouvrières pour lesquelles les groupes de potagers sont édifiés. C'est là également que cette institution à but non lucratif trouve des terrains non seulement abordables à la location ou à l'achat mais encore accessibles par le réseau de tramways, en plein essor. Par ailleurs, grâce au cadre campagnard de ces portions du territoire, l'Cuvre des jardins de famille cherche à éloigner ses bénéficiaires des méfaits supposés de leur environnement urbain (fig. 1). Toutefois, rien ne semble plus important pour la localisation des jardins ouvriers que la présence ou du moins la proximité d'une source régulière d'approvisionnement en eau : sources, cours d'eau (le ruisseau des Aygalades, la rivière du Jarret et le fleuve côtier de l'Huveaune) ou, plus généralement, le canal de Marseille qui achemine, depuis le $\mathrm{XIX}^{\mathrm{e}}$ siècle, les eaux de la Durance dans toutes les banlieues agricoles et permet un système d'irrigation gravitaire indispensable aux cultures.

Figure 1

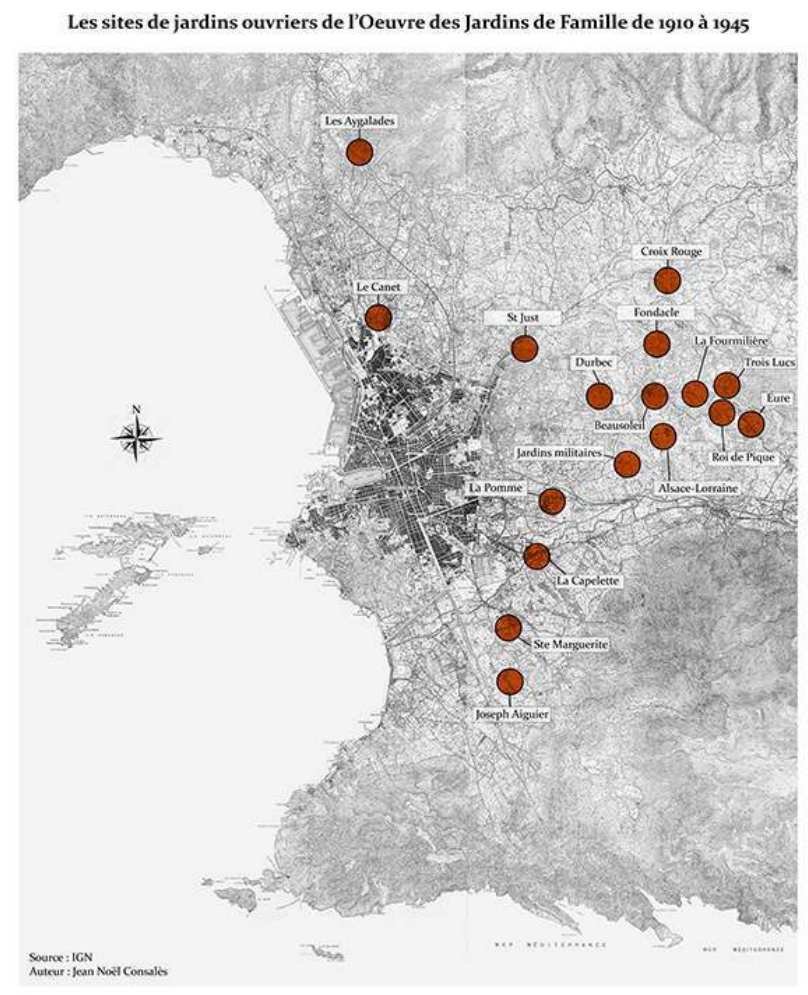

Les sites de jardins ouvriers de l'CEuvre des jardins de famille de 1910 à 1945.

(c) IGN (c) Jean Noël Consalès. 
des nait, au début du Xx siecle, le mouvement de la LFCTF est relayé à l'échelle locale par des notables catholiques, philanthropes et hygiénistes. Ainsi, à Marseille, l'ÆEuvre des jardins de famille est-elle créée, en 1910, sous l'impulsion de Joseph Aiguier, directeur de la section marseillaise de la Caisse régionale d'assurances agricoles mutuelles contre l'incendie. Fervent catholique, il participe à La Provence coopérative, organisme "d'études et de réalisations d'œuvres sociales» (registre de l'œuuvre des jardins de famille, 5 août 1910). Celle-ci possède plusieurs divisions auxquelles Joseph Aiguier, influencé par la propagande de l'abbé Lemire, propose d'ajouter une institution de jardins ouvriers. Le 30 janvier 1910, l'ÆEuvre des jardins de famille est donc officiellement fondée. Elle n'est encore qu'une section de La Provence coopérative, dont elle ne se démarque qu'en 1912. Appel est fait auprès de quelques notables pour constituer le comité de l'œEuvre. Ce dernier est très vite formé, grâce à la participation de médecins, d'avocats, d'industriels et d'ingénieurs, favorables aux théories hygiénistes. Un président d'honneur est trouvé en la personne du poète Frédéric Mistral, partisan du retour à la terre. Celui-ci donne à l'ÆEuvre sa devise : «Quau jouine planto, viei canto!» («Qui plante jeune, chante vieux!»). Désormais, l'institution existe mais elle n'a ni terrains, ni jardiniers, ni encore de statuts précis. Après avoir visité, en vain, 28 terrains, les membres de l'đEuvre s'intéressent à une $29^{\mathrm{e}}$ parcelle. Celle-ci est située dans le lieu-dit de Fondacle, entre les noyaux villageois de Saint-Julien et des Olives, au cœur de la banlieue agricole. Le bail est fixé avec le propriétaire (un agriculteur) et, au mois d'août 1911, 30 lopins cultivables sont attribués gratuitement à des familles nombreuses soigneusement choisies par le comité de l'Euvre.

En 1912, l'ÆEuvre devient une association indépendante. Elle adhère à la Ligue française $\mathrm{du}$ coin de terre et du foyer et se dote de statuts. Elle se fixe pour but de favoriser la famille, au point de vue matériel, moral et hygiénique, en lui procurant par le prêt gratuit d'un jardin à cultiver :

- un supplément de ressources dans le ménage,

12 - un encouragement à la prévoyance et à l'épargne,

13 - un moyen de lutter efficacement contre l'alcoolisme,

14 - une distraction saine,

15 - un plaisir que toute la famille partage,

16 et une sorte de sanatorium, où les parents viennent avec leurs enfants respirer librement au grand air et faire provision de santé ${ }^{15}$.

17 Pour les dirigeants de l'association, «les jardins de famille sont des jardins ouvriers perfectionnés où la culture de la terre marche de pair avec les œuvres sociales ${ }^{16}$.

18 Grâce à cette structuration, l'association consent, en 1913, à la location d'un nouveau terrain dans le faubourg des Trois-Lucs, aux confins de l'actuel territoire communal. Bientôt, vingt jardiniers y sont installés.

19 Avec les deux guerres mondiales et la crise économique des années 1930, les initiatives associatives en matière de jardins ouvriers sont progressivement encouragées, voire relayées par les collectivités territoriales, notamment dans le cadre du retour à la terre prôné par le régime de Vichy. Réclamés par une population lésée par les rationnements, les potagers urbains, recentrés sur leur vocation nourricière, deviennent un élément important de l'amélioration des conditions de vie. À Marseille, dès le début de la Première Guerre mondiale, l'œEuvre des jardins de famille est ainsi sollicitée pour 
accroitre le nombre de parcelles potagères qui, dans ce contexte de conflit, se chargent de valeurs patriotiques. Multipliant ses créations, l'association se concentre alors sur deux types d'actions :

20 - La mise en place et l'aide à la gestion de jardins militaires. À partir de 1916, cette institution participe, en effet, à la bonne marche de deux groupes (dont un situé entre les noyaux villageois de la Pomme, de Saint-Barnabé et des Caillols), destinés aux soldats démobilisés. Elle réserve, en outre, dans ses propres ensembles, des potagers pour les veuves de guerre.

21 - Le développement des jardins ouvriers. De nombreux collectifs de potagers sont établis à l'est de la ville, grâce à des locations, dans les faubourgs des Caillols (groupe «Alsace Lorraine » créé en 1915, 21 lots), de la Croix-Rouge (groupe Croix-Rouge, 8 lots, en 1915) et surtout de Saint-Julien (en 1916, le groupe Durbec, 15 lots, qui disparait dès 1919, le groupe « Beausoleil », qui disparaît dès 1918 et le groupe de la Fourmilière, 15 parcelles).

22 Entre les deux guerres, l'œeuvre des jardins de famille développe, diversifie et adapte ses interventions en fonction des contraintes et des opportunités foncières. Progressivement, les règles d'aménagement et les schémas d'organisation s'affinent. Suivant un plan orthogonal, les ensembles créés à cette époque se composent le plus souvent de deux sections d'une vingtaine de parcelles, alignées autour d'une allée centrale. La forme des groupes de jardins ouvriers se standardise. En 1920, à la demande d'une autre association de bienfaisance, l'Assistance par le travail, l'CEuvre récupère la gestion de quatre groupes de jardins ouvriers dans les faubourgs de Sainte-Marguerite (48 lots), de la Pomme (46 lots) des Aygalades (43 lots) et de la Capelette (18 lots). Par ailleurs, sur un terrain mis à disposition gratuitement, elle crée au Canet un nouveau site de 110 parcelles, inauguré par l'abbé Lemire le 3 septembre 1922. Mais en 1924, le groupe des Trois-Lucs et celui de la Fourmilière (faubourg de Saint-Julien) disparaissent car les terrains sont vendus par leurs propriétaires. Il en va de même de 1930 à 1938 pour les ensembles "AlsaceLorraine » (faubourg de Saint-Julien), du Canet, de la Capelette et de Sainte-Marguerite. La disparition de ces collectifs de potagers est néanmoins compensée, pour la première fois, par une politique d'achat. Trois terrains sont ainsi acquis. Deux sont implantés, à l'est, dans les faubourgs des Trois-Lucs et de la Valentine (groupe du Roi de Pique et groupe de l'Eure), l'autre, au sud, à Mazargues. Baptisé « Joseph Aiguier » en hommage au créateur de l'œuvre, mort en 1938, ce groupe de 110 lots est divisé en trois sous-entités (Mazargues, Pinède et Cabot). Dès son origine, cet ensemble se dote de "cures d'air »: parcelles non jardinées et munies d'un cabanon, uniquement destinées à la villégiature. Durant la Seconde Guerre mondiale, l'Cuuvre des jardins de famille se contente de gérer ses acquis, ne sollicitant que la création d'un petit groupe de 15 parcelles, à Saint-Just, qui disparaît dès 1947. Déstabilisée par la disparition de Joseph Aiguier, l'association semble tourner au ralenti et rester à l'écart de la grande vague de créations qui intervient, à Marseille, à partir de la loi en faveur des jardins ouvriers instaurée le 18 août 1940 par le gouvernement de Vichy. C'est à cette époque que sont créés, par exemple, les « jardins du Maréchal » (actuels jardins du Castellas), immense site de plus de 240 parcelles entre les quartiers de Saint-Joseph et celui des Aygalades, au nord de la commune.

23 Mais durant les Trente Glorieuses, le processus d'urbanisation massive qui gagne la France fait disparaître près de $80 \%$ des jardins ouvriers du territoire national ${ }^{17}$. Réduits au rang de simples réserves foncières ou de terrains à bâtir, ceux-ci apparaissent, le plus souvent, comme obsolètes, gênants et totalement incompatibles avec les nouveaux canons de la modernité. Suivant cette évolution, la loi du 26 juillet $1952^{18}$ rebaptise avec 
un terme beaucoup moins connoté socialement les groupes de potagers urbains. Désormais, les jardins ouvriers deviennent des jardins « familiaux ». À Marseille, bien que difficilement quantifiable, le déclin touche essentiellement les groupes de potagers créés à titre précaire durant la Seconde Guerre mondiale. À partir des années 1950, la pression foncière devient ainsi un sujet récurrent des registres de l'œuvre des jardins de famille. Trois groupes (Aygalades, Joseph Aiguier et Roi de Pique) sont directement menacés de disparition par l'urbanisation galopante de banlieues désormais bien plus résidentielles qu'agricoles. En 1968, le site du Roi de Pique finit d'ailleurs par être absorbé par un aménagement routier. Pour compenser cette perte, l'association acquiert un terrain dans le quartier des Trois-Lucs et aménage le groupe de Berre-Pastour (39 parcelles). Inquiète de la situation foncière de plus en plus menacée de ses sites, l'association adresse tout de même, le 16 octobre 1970, une véritable lettre de doléances au maire de Marseille :

[...] L'CEuvre des jardins de famille, ayant un but non lucratif et étant tenue de maintenir son patrimoine foncier au profit des familles bénéficiaires, demande à l'expropriant de lui attribuer des sommes compensatoires de la valeur des terrains perdus afin d'en acheter d'autres. Mieux encore, elle demande que le dit expropriant lui procure d'autres terrains en compensation de cette valeur dans une banlieue accessible pour lui permettre de poursuivre son but qui est de procurer aux travailleurs la jouissance d'un lopin de terre pour cultiver les légumes nécessaires à la consommation familiale et pour lui permettre de venir en famille respirer le bon air $[. . .]^{19}$.

24 Très répandu au sein des associations affiliées à la LFCTF, ce type de discours devient une véritable ligne de combat fédératif qui aboutit, le 10 novembre 1976, à la promulgation de la loi relative à la création et à la protection des jardins familiaux. Précisant qu'en cas d'expropriation à des fins d'utilité publique, les groupes de potagers doivent être replacés par l'expropriant en surfaces et en équipements équivalents ${ }^{20}$, celle-ci incite les municipalités à agir avec une grande prudence en matière de potagers urbains.

De fait, à partir de cette époque, les rapports qu'entretiennent les villes avec leurs jardins familiaux varient fortement en fonction des contextes locaux. Ainsi, à Marseille, la situation des jardins familiaux semble ambivalente, voire ambiguë ${ }^{21}$. D'une part, en effet, la pression foncière reste constante pour de nombreux groupes situés de longue date sur des emplacements réservés. La contrainte est telle que l'œEuvre des jardins de famille décide, en 1994, d'instaurer la dévolution de ses terrains au profit de l'organisme le plus habilité à garantir la pérennité des jardins familiaux marseillais: la Ligue française du coin de terre et du foyer-Fédération nationale des jardins familiaux. Pour ce faire, l'œuvre des jardins de famille est dissoute et remplacée par le comité local de Marseille de la Ligue, sorte de succursale, à l'échelle locale, de la Fédération nationale. Parallèlement, sur les terrains en simple location (groupe de la Pomme disparu et transféré sur le site de Montolivet et groupe des Aygalades), des associations de gestion indépendantes sont créées. D'autre part, au début des années 2010, la Ville de Marseille se dote de personnels dédiés aux jardins collectifs et accompagne la création d'un groupe (Les Jardins du théâtre Athéna, 63 parcelles) dans le quartier de Château-Gombert, au nord-est de la ville. Elle prévoit, en outre, l'établissement de deux nouveaux sites dans le cadre de l'aménagement la rocade L2. Ce sont donc en tout près de 900 parcelles de jardins familiaux, aux épaisseurs historiques variées, qui se répartissent aujourd'hui de manière assez équilibrée au sein de la périphérie de Marseille (fig. 2). 
Figure 2

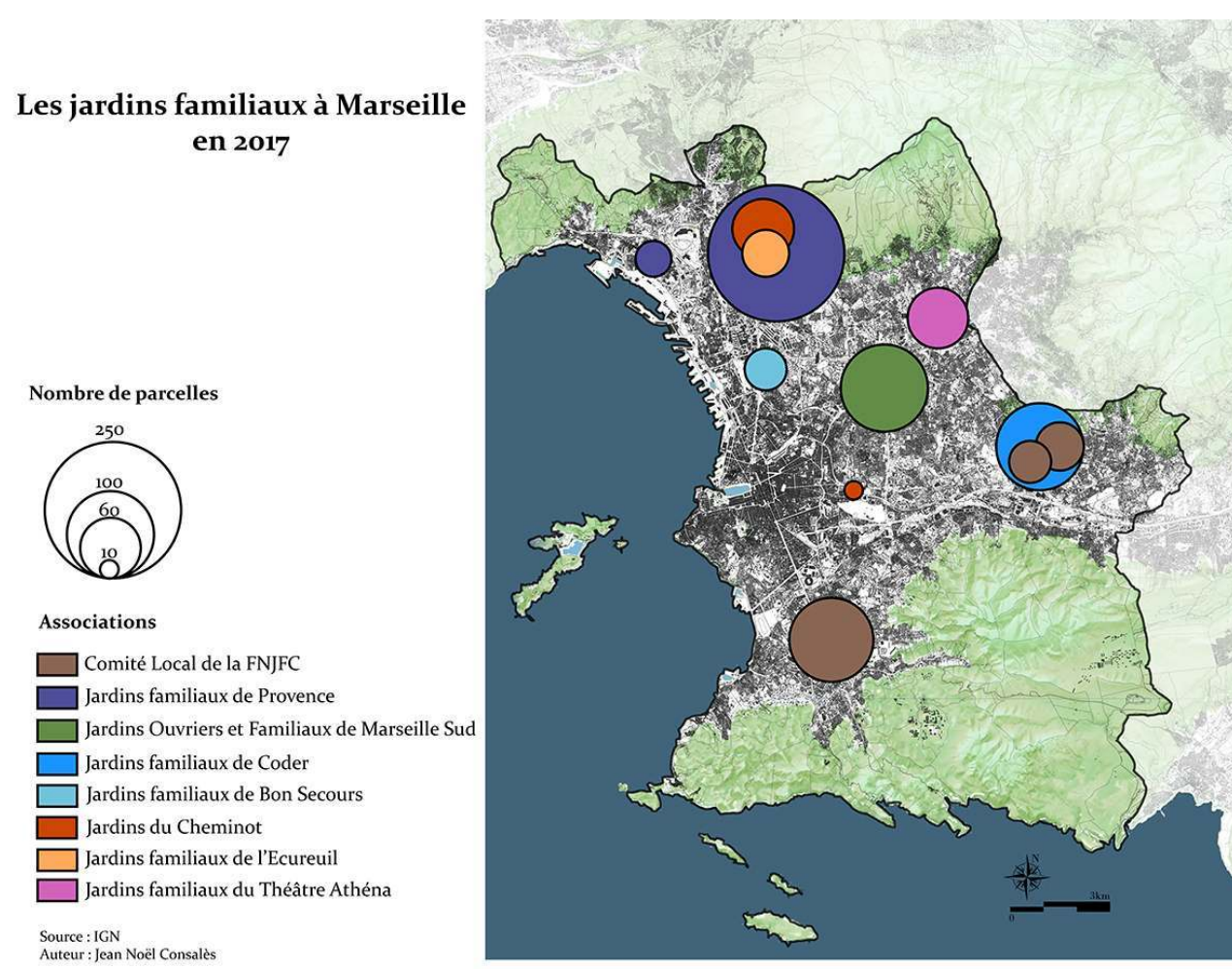

Les jardins familiaux à Marseille en 2017.

(c) IGN (c) Jean Noël Consalès.

\section{Des jardins partagés de la périphérie au jardinage des rues du centre-ville}

En France, parallèlement à l'évolution fluctuante des jardins familiaux, de nombreuses formes dérivées de collectifs de potagers et de potagers collectifs se sont développées au cours des années 1990. Définis comme « les jardins créés ou utilisés en vue de favoriser la réintégration des personnes en situation d'exclusion ou en difficulté sociale ou professionnelle [...] $»^{22}$, les « jardins d'insertion » sont ainsi apparus en réaction aux effets structurels de la crise économique que connaît le pays depuis la fin des Trente Glorieuses. Faisant écho aux community gardens qui se développent en Amérique du Nord depuis les années $1970^{23}$, les jardins partagés se présentent, pour leur part, comme «les jardins créés ou animés collectivement, ayant pour objet de favoriser des liens sociaux de proximité par le biais d'activités sociales, culturelles ou éducatives et étant accessibles au public $\aleph^{24}$. Témoignant de dynamiques sociales de plus en plus prégnantes ${ }^{25}$, leur important essor au cours des deux dernières décennies s'appuie sur des institutions organisées qui savent monter des projets cohérents, mobiliser les décideurs et débloquer les financements. Nombre de ces associations fonctionnent en réseau et sont notamment regroupées, à l'échelle nationale, au sein du collectif $«$ Jardin dans tous ses états ${ }^{26}$. Cette montée en puissance des jardins partagés doit encore beaucoup à la diffusion de l'expérience mise en place par la mairie de Paris $^{27}$ qui, à bien des égards, sert de référence, voire de modèle, à de nombreuses communes. 
27 À Marseille, le développement des jardins partagés commence, en 1995, avec la création de l'association des Jardins d'Amélie (Aménagement d'un lieu pour une insertion par l'échange) qui, dès le départ, se construit en opposition avec le modèle des jardins familiaux. Elle reproche en effet à ces derniers « de souffrir d'une réglementation rigide et d'un enfermement sur eux-mêmes, ainsi que d'être parfois inexistants dans la vie du quartier où ils sont implantés $»^{28}$. Contre cela, elle propose l'édification de $"$ jardins solidaires [...] permettant de retisser des relations, retrouver des espaces de concertation et de participation et de favoriser la mixité sociale, intergénérationnelle et culturelle $»^{29}$. Partant de ce principe, elle crée, en 1997, les jardins des Dènes (Denrées pour une économie nouvelle et solidaire), initiative sise entre le jardin d'insertion et le jardin partagé. Pour ce faire, elle utilise un terrain municipal de $1350 \mathrm{~m}^{2}$ ouvert sur le quartier populaire de la Belle de Mai. En 1999, les jardins d'Amélie se positionnent comme l'organisme de coordination du réseau des Jardins solidaires méditerranéens ${ }^{30}$ (RJSM) qui regroupe de nombreuses expériences de jardins partagés dans le sud-est de la France ${ }^{31}$. Fondé sur une gouvernance horizontale, celui-ci s'impose progressivement comme une plaque tournante des initiatives marseillaises en faveur des jardins partagés. Deux structures adhérentes au RJSM, le PADES (programme autoproduction et développement social) ${ }^{32}$ et l'AMIEU (l'atelier marseillais d'initiatives en écologie urbaine) ${ }^{33}$ se démarquent alors en se spécialisant dans le portage, l'accompagnement et le suivi de nombreux projets de jardins partagés. Au fil du temps, ces derniers tendent à évoluer. Si l'esprit de communauté reste le fondement de l'action, les formes d'établissement peuvent varier. En fonction des attentes des jardiniers, l'usage collectif d'une parcelle unique laisse souvent la place à des appropriations individuelles d'un site parcellisé et fermé. De fait, bien que leurs surfaces soient beaucoup plus restreintes (aux alentours de $1000 \mathrm{~m}^{2}$ en moyenne), les jardins partagés marseillais prennent généralement la forme de jardins familiaux.

À partir du milieu des années 2000, les jardins partagés ne cessent donc de croître, portés par des initiatives interprétant une demande sociale sans cesse renouvelée à l'égard d'espaces urbains jardinés. Les particularités de la population marquée par d'énormes disparités et inégalités socio-économiques, par un taux de chômage de 18,5\% (contre $10,4 \%$ en moyenne dans les autres villes françaises) ou par un taux de pauvreté de $25,5 \%$ (contre 14,3\% en moyenne en France) renforcent les besoins exprimés à l'échelle locale. À cet égard, de nombreux projets de création et d'animation de jardins partagés se situent dans des quartiers prioritaires de la politique de la ville et bénéficient du soutien des équipes du contrat de ville. Mais la dynamique marseillaise en faveur des jardins partagés s'explique aussi par les potentialités géographiques de l'immense territoire communal caractérisé, dans sa périphérie, par de nombreuses opportunités foncières : des friches et des délaissés propices tant aux appropriations citoyennes qu'à l'urbanisation. Placés en dehors du droit qui protège, depuis 1976, les jardins familiaux de la pression foncière, les jardins partagés apparaissent comme des formes souples d'occupation des terrains ${ }^{34}$. Ils permettent ainsi de développer des projets sans pour autant figer la vocation productive des sols ${ }^{35}$. Dans un contexte d'intense spéculation, cette caractéristique rassure les propriétaires qui consentent plus facilement à signer des baux ou des conventions de mise à disposition, plus ou moins précaires, avec les groupes de jardiniers. Ainsi, en 2010, le service des Espaces verts et de la Nature (SEVN) de la commune initie la charte des jardins partagés de Marseille pour allouer gratuitement, par la contractualisation, des terrains communaux à des « collectifs d'habitants responsables de la dynamique du jardin 
et des pratiques de jardinage écoresponsables $»^{36}$. Parallèlement, les bailleurs tendent à intégrer des projets de potagers aux plans de valorisation des espaces délaissés de leurs parcs d'habitat social. Les aménageurs privés, eux-mêmes, pensent à inclure des jardins partagés dans leurs programmes immobiliers, notamment au sein des opérations qui possèdent une certaine dimension environnementale ${ }^{37}$.

Placée entre des attentes sociales prégnantes, des réponses d'acteurs de plus en plus structurées et des possibilités foncières incontestables, la diffusion constante des jardins partagés sur le territoire marseillais reste inféodée à des logiques d'implantation précises. La répartition spatiale des quelque 53 sites recensés (fig. 3) détermine ainsi une géographie dominée par deux facteurs fortement corrélés: la pauvreté et le mode d'habiter des populations. De fait, les jardins partagés marseillais ont une vocation sociale certaine qui induit des emplacements privilégiés. Au cœur des quartiers populaires centraux et péricentraux ( $2^{\mathrm{e}}$ et $3^{\mathrm{e}}$ arrondissements de Marseille), ils se greffent sur des dents creuses sises au sein d'un tissu urbain dense caractérisé par un habitat ancien, fortement dégradé, occupé par des résidents très pauvres (le taux de pauvreté oscille entre $44 \%$ et $55 \%$ ). En périphérie, dans les quartiers nord ( $14^{\mathrm{e}}$ et $15^{\mathrm{e}}$ arrondissements) et sud-est (le long de la vallée industrielle du fleuve côtier de l'Huveaune), ils se fixent préférentiellement au pied des immeubles des grands ensembles (voir fig. 3) habités par des populations pauvres (le taux de pauvreté oscille ici entre $20 \%$ et $44 \%$ ). Bien que partagés, ces jardins n'en demeurent pas moins parcellisés. Les lopins (voir fig. 4) sont généralement petits (de 10 à $25 \mathrm{~m}^{2}$ en moyenne) mais nombreux (de 20 à 90 parcelles par jardin), ce qui témoigne de l'engouement local pour le jardinage individuel et l'autoproduction.

Figure 3

Les jardins partagés à Marseille en 2017

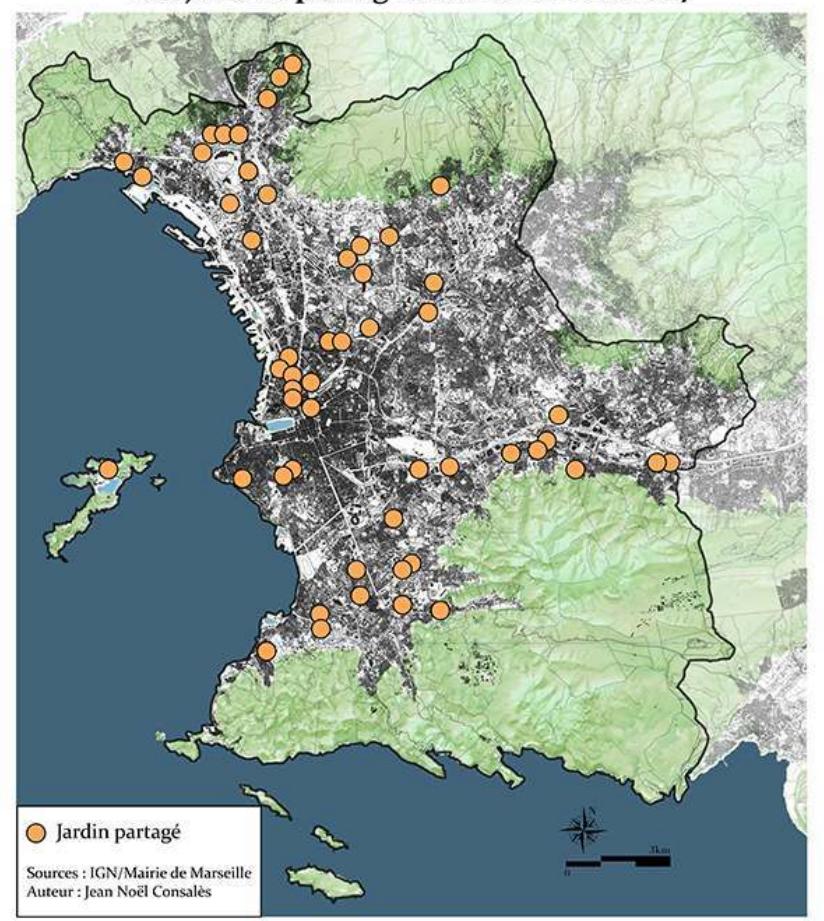

Les jardins partagés à Marseille en 2017.

(c) IGN @ Jean Noël Consalès. 
Cet attrait populaire de plus en plus marqué transparaît encore dans la récente végétalisation de certaines rues du centre-ville de Marseille. S'inscrivant dans une tendance quasi planétaire qui relie le mouvement américain de green guerilla ${ }^{38}$, celui des « incroyables comestibles ${ }^{39}$ » né en Angleterre en 2008 ou encore celui de " renaturation » instauré, au début des années 2000, par certaines villes françaises (Rennes et Lyon), cette dynamique consiste en une appropriation jardinière de l'espace public par un collectif d'habitants. À travers cette action citoyenne, ces derniers entendent "reconquérir les rues $»^{40}$ en disposant le long de la voirie des pots et des bacs de culture garnis de divers végétaux parmi lesquels figurent parfois des plantes potagères. Si cette nouvelle pratique est née au début des années 2000 sur le boulevard Honorine (dit " rue des Pots-bleus ») dans le quartier périphérique des Crottes au nord de Marseille, très vite elle s'est diffusée au fil des rues du centre-ville. Les quartiers populaires de Noailles (les rues de l'Arc et Sénac-de-Meilhan qui sont investies dès 2010-2011 comptent aujourd'hui plus de 150 jardinières chacune), du Panier, de Belsunce, du Chapitre, de Saint-Charles et du Camas témoignent le plus de cette végétalisation (fig. 4) ${ }^{41}$. Le processus de propagation est tel qu'il oblige la mairie de Marseille à se doter, en 2015, d'un permis de végétaliser nommé visa vert et d'une charte de végétalisation de l'espace public marseillais. Ces documents entendent «permettre aux particuliers d'occuper temporairement et gratuitement l'espace public par l'installation de plantes tout en respectant la sécurité et l'utilisation des voies par les autres usagers (personnes à mobilité réduite, pompiers...) $»^{42}$ . Ils se présentent donc comme une tentative, souvent vaine, de contrôler une dynamique jardinière enclenchée par des habitants désireux d'améliorer leur cadre de vie et de favoriser les sociabilités à l'échelle de la rue.

Figure 4

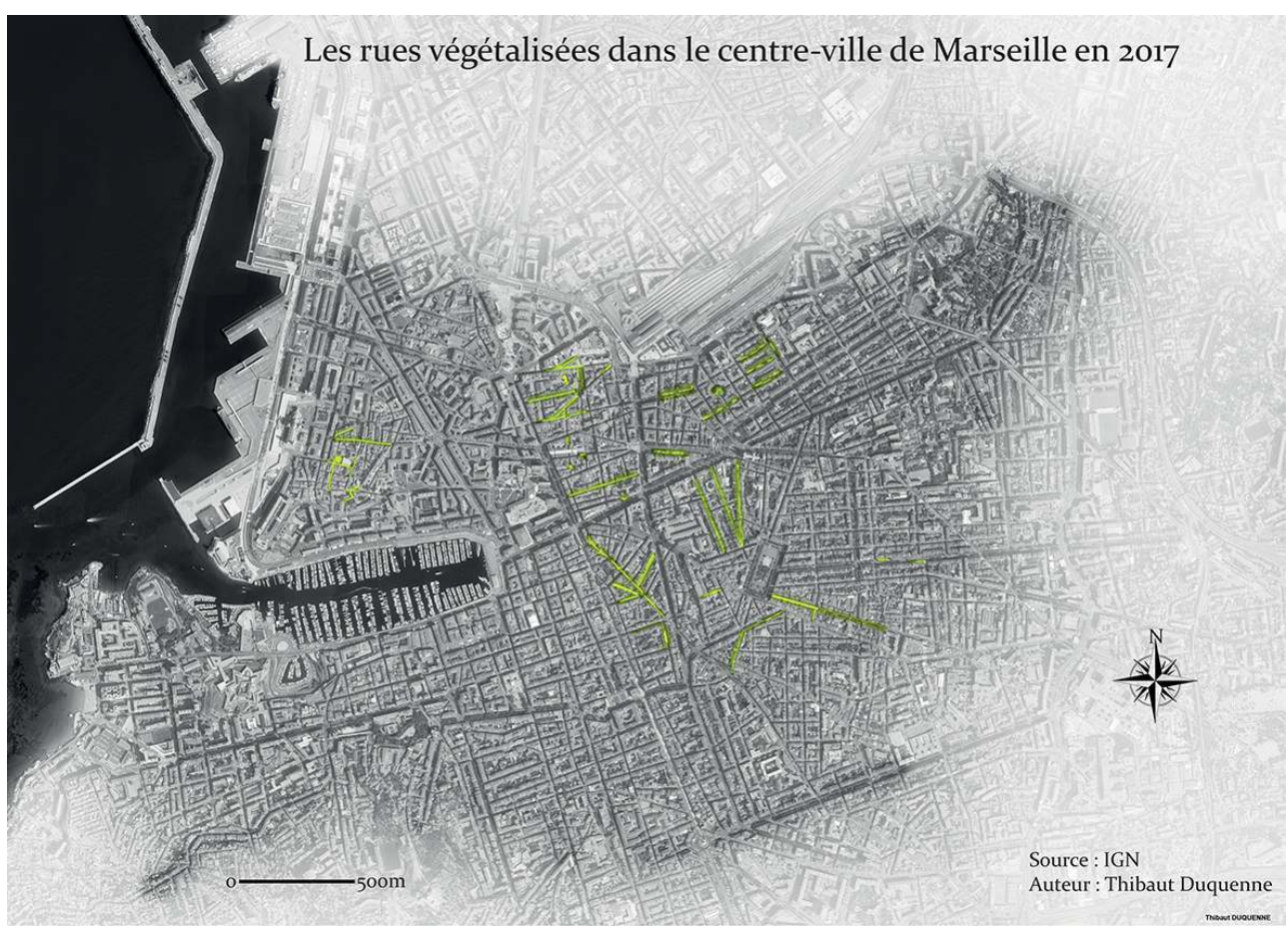

Les rues végétalisées dans le centre-ville de Marseille en 2017.

(c) IGN (Thibaut Duquenne. 


\section{Conclusion : Quelle portée patrimoniale pour les jardins collectifs marseillais?}

$31 \mathrm{Au}$ terme de la démarche géohistorique proposée, il convient très certainement de reconnaître la valeur d'exemple du cas marseillais. Rares sont en effet les communes au sein desquelles il est possible de dénombrer autant de formes différentes de jardins collectifs issus de phases de production aussi distinctes. Ainsi, parmi les douze groupes de jardins familiaux présents sur le territoire, trois datent d'avant 1939. À cette époque, l'essor des jardins ouvriers s'exprime, dans la banlieue agricole, grâce à l'investissement d'œuvres catholiques, philanthropiques et hygiénistes influencées par l'abbé Lemire et sa LFCTF. Six autres groupes sont directement hérités de la Seconde Guerre mondiale, période durant laquelle le régime de Vichy encourage fortement les créations. Deux groupes résultent encore des logiques de compensation qu'instaure, en 1976, la loi en faveur des jardins familiaux, tandis que s'urbanise massivement la banlieue de Marseille. Enfin, un groupe est une production récente, portée par le travail conjoint d'une association d'habitants et des services de la mairie. Par ailleurs, avec le jardin des Dènes, la commune accueille, depuis 1995, l'une des plus anciennes expériences de jardin partagé en France. Celle-ci a ouvert la voie aux 52 autres initiatives de ce type qui, selon l'Union nationale des entreprises du paysage (UNEP) et Hortis (l'association des responsables d'espaces nature en ville), mettent Marseille à la seconde place des villes françaises les mieux dotées en jardins partagés, en $2016^{43}$. Ceux-ci ne cessent en effet de se développer, tant dans la périphérie que dans le centre, où s'inventent également de nouvelles dynamiques de jardinage de rue. Si toutes les formes de jardins collectifs semblent donc, en apparence, de mieux en mieux reconnues par les acteurs du territoire, notamment pour leurs fonctions sociales, économiques et environnementales ${ }^{44}$, force est de constater que leur dimension patrimoniale reste encore largement ignorée. De fait, l'épaisseur historique des sites et des pratiques sociales qui leur sont associées n'entre jamais en considération dans la gestion urbaine de ce type d'espace. Les groupes de jardins familiaux les plus anciens restent ainsi les plus précarisés par la pression foncière. L'exemple du jardin Joseph Aiguier à Mazargues est, à ce titre, particulièrement éloquent. Menacé d'amputation partielle et de restructuration complète par l'avancée projet du boulevard urbain Sud (B.U.S.), ce groupe, hérité de 1930, est un sujet d'inquiétude pour ses riverains et ses jardiniers. Depuis plusieurs années, ces derniers tentent de faire valoir le statut patrimonial d'un site qu'ils souhaiteraient voir rester en l'état ${ }^{45}$. Pour ce faire, ils arguent de : 


\section{NOTES}

1. - L'auteur tient ici à remercier vivement pour leur aide amicale les paysagistes-urbanistes Brice Dacheux-Auzière, Thibaut Duquenne et Alain Millias.

2. - Proposition de loi relative aux jardins collectifs adoptée en première lecture par le Sénat le 14 octobre 2003 mais bloquée à l'Assemblée nationale.

3. - Par géohistoire, il faut entendre ici l'étude de la répartition d'un phénomène spatial (en l'occurrence, les jardins collectifs) sur le temps long.

4. - Il s'agit de trois volumes consignant les comptes rendus des assemblées générales de cet organisme, de 1910 à 1975, et de deux mémorandums détaillant ses activités entre 1921 et 1965.

5. - La Fédération nationale des jardins familiaux et collectifs, anciennement nommée Ligue française du coin de terre et du foyer, a été créée en 1896.

6. - Les archives de la FNJFC ainsi que celles du département des Bouches-du-Rhône et de la Ville de Marseille fournissent de maigres renseignements quant à la création d'autres associations marseillaises de jardins ouvriers. L'inventaire des groupes de potagers implantés sur la commune demeure donc partiel. Par ailleurs, lorsqu'elles existent, les informations disponibles restent très imprécises sur la localisation, le nombre exact de parcelles et le devenir des jardins. Dans ce contexte, il est impossible de prétendre à l'exhaustivité.

7. - CONSALÈS, Jean Noël. Les Jardins familiaux à Marseille. Diplôme d'études approfondies en géographie et aménagement du territoire. Aix-en-Provence : Université de Provence, 1998.

8. - CONSALÈS, Jean Noël. Les Jardins familiaux à Marseille, Gênes et Barcelone : laboratoires territoriaux de l'agriculture urbaine dans l'Arc Méditerranéen. Thèse de géographie et aménagement du territoire. Aix-en-Provence : Université de Provence, 2004.

9. - ANR (Agence nationale pour la recherche) -12-VBDU-0011, Jardins associatifs urbains.

10. - L'auteur est administrateur de la FNJFC et a été président de son comité local à Marseille.

11. - Le terrianisme est une doctrine créée en 1892 par un groupe de démocrates-chrétiens du Nord afin de défendre et encourager la petite propriété insaisissable et les valeurs terriennes.

12. - Art. L. 561-1. de la proposition de loi relative aux jardins collectifs du 14 octobre 2003. Il convient de signaler deux études sur Marseille produites dans le cadre de Jassur: DACHEUXAUZIÈRE, Brice. Les Jardins partagés comme projet de paysage. Acteurs, gouvernance et processus de mise en œuvre des jardins associatifs urbains à Marseille. M2 Urbanisme. Marseille: Aix-Marseille Université, 2014 et MILLIAS, Alain. Le Jardin partagé: un jardin comme les autres. Territoires et gouvernance des jardins associatifs urbains à Marseille. M2 Urbanisme. Marseille: Aix-Marseille Université, 2014.

13. - CABEDOCE, Béatrice et PIERSON, Philippe. Cent ans d'histoire des jardins ouvriers ; 1896-1996, La Ligue française du coin de terre et du foyer. Grane : Créaphis, 1996.

14. - Littéralement «terroir » en provençal. Ce terme désigne, à l'échelle locale, l'ensemble des banlieues agricoles inscrites sur le territoire de Marseille.

15. - Registre de l'œuuvre des jardins de famille, statuts d'association loi 1901, adoptés le 21 juin 1912, Titre 1 , article $\mathrm{n}^{\circ} 1$.

16. - Registre de l'œuuvre des jardins de famille, statuts d'association loi 1901, adoptés le 21 juin 1912, Titre 1 , article $\mathrm{n}^{\circ} 1$.

17. - CABEDOCE, Béatrice et PIERSON, Philippe. Op. cit.

18. - Loi $\mathrm{n}^{\circ} 52-895$ du 26 juillet 1952 portant codification de la législation des jardins familiaux.

19. - Registre de l'œuuvre des jardins de famille, 16 octobre 1970. 
20. - Article 2 de la loi $\mathrm{n}^{\circ} 76-1022$ du 10 novembre 1976 relative à la création et à la protection des jardins familiaux

21. - CONSALÈS, Jean Noël. «Les jardins familiaux marseillais : laboratoires territoriaux d'une agriculture urbaine en Méditerranée ». Méditerranée, 2000, n 95, 3-4, « Dynamiques spatiales des cultures spéciales», p. 81-88. Voir sur le site: https://www.persee.fr/doc/ medit_0025-8296_2000_num_95_3_3179 [consulté le 19/10/2018].

22. - Art. L. 561-1. de la proposition de loi relative aux jardins collectifs du 14 octobre 2003.

23. - BAUDRY, Sandrine. «Les community gardens de New York City : de la désobéissance civile au développement durable ». Revue française d'études américaines, 2011/3, n 129, « De la nature à l'environnement ", p. 73-86. Voir sur le site : https://www.cairn.info/revue-francaise-d-etudesamericaines-2011-3-page-73.htm [consulté le 19/10/2018].

24. - Art. L. 561-1. de la proposition de loi relative aux jardins collectifs du 14 octobre 2003.

25. - DEMAILLY, Kaduna-Ève. Jardiner les vacants. Fabrique, gouvernance et dynamiques sociales des vacants urbains jardinés du nord-est de l'île-de-France. Thèse de géographie. Paris : université Paris-1, 2014.

26. - Voir le site : http://jardins-partages.org [consulté le 19/10/2018].

27. - Datant de 2003, le programme «Main verte» encourage, accompagne et coordonne les jardins partagés parisiens.

28. - ALPHANDÉRY-FABRELLO, Paola. Vers des jardins citoyens! Une expérience en cours. DESS de sciences économiques. Aix-en-Provence : université d'Aix-Marseille, 1996.

29. - Ibid.

30. - Voir le site : http://www.reseaujsm.org [consulté le 19/10/2018].

31. - Le RJSM est membre du JTSE.

32. - Voir le site : https://www.padesautoproduction.net [consulté le 19/10/2018].

33. - Voir le site: http://jardinspourtous.amieu.over-blog.com/pages/ PRESENTATION_DE_LASSOCIATION-7251270.html [consulté le 19/10/2018].

34. - Cette situation fait écho à celle décrite par Arnaud Frauenfelder, Christophe Delay et Laure Scalambrin pour le cas de Genève. (FRAUENFELDER, Arnaud, DELAY, Christophe et SCALAMBRIN, Laure. "Potagers urbains vs jardins familiaux ? Réforme urbaine et controverses autour du beau jardin et son usage légitime ». Espaces et Sociétés, 2014/3, nº 158, « Agriculture et ville », p. 67-81).

35. - CONSALÈS, Jean Noël. «Les jardins collectifs à Marseille. Quelles relations entre la ville et l'agriculture urbaine ?». Dans DONADIEU, Pierre (dir.). L'Agriurbanisation : rêves ou réalités ? Paris : Editopics, 2014, p. 115-122.

36. - Voir sur le site: http://environnement.marseille.fr/sites/default/files/contenu/ environnement/charte_jardins_partages.pdf [consulté le 19/10/2018].

37. - Ceci fait écho à la situation bordelaise décrite par Nicolas D’Andrea et Pascal Tozzi (D'ANDREA, Nicolas et TOZZI, Pascal. «Jardins collectifs et écoquartiers bordelais : De l'espace cultivé à un habiter durable?». Norois, 2014, $\mathrm{n}^{\circ} 231$, «Les modes d'habiter à l'épreuve de la durabilité », p. 61-74). Voir le site : https://journals.openedition.org/norois/5087 [consulté le 19/10/2018].

38. - Voir le site : http://www.greenguerillas.org [consulté le 19/10/2018].

39. - Voir le site : https://www.incredible-edible-todmorden.co.uk [consulté le 19/10/2018].

40. - SOULIER, Nicolas. Reconquérir les rues. Exemples à travers le monde et pistes d'actions. Paris : Ulmer, 2012.

41. - DUQUENNE, Thibaut. La Végétalisation dans les espaces intra-urbains denses. M2 Urbanisme. Marseille : Aix-Marseille Université, 2017.

42. - Voir le site: http://environnement.marseille.fr/nature-en-ville/vegetalisation-des-rues [consulté le 19/10/2018].

43. - Voir le site : http://www.lesentreprisesdupaysage.fr/actualites/2016-r\%C3\%A9sultats-delenqu\%C3\%AAte-de-lobservatoire-des-villes-vertes-jardin-2-0 [consulté le 19/10/2018]. 
44. - CONSALÈS, Jean Noël. Les Jardins familiaux à Marseille, Gênes et Barcelone: laboratoires territoriaux de l'agriculture urbaine dans l'Arc méditerranéen. Thèse de géographie et aménagement du territoire. Aix-en-Provence : université de Provence, 2004.

45. - Voir le site : http://jardinaiguier.canalblog.com [consulté le 19/10/2018].

\section{RÉSUMÉS}

Fondé sur l'analyse d'archives historiques et sur un suivi de terrain au long cours, cet article propose une lecture géohistorique des jardins collectifs à Marseille. Il identifie les idées qui, de l'échelle nationale à l'échelle locale, génèrent leurs évolutions, les acteurs qui portent leurs dynamiques et les espaces urbains qui leur sont dédiés. Dans une première partie, il étudie le processus territorial qui a conduit les jardins ouvriers sis dans les faubourgs agricoles du début $\mathrm{du} \mathrm{xx}^{\mathrm{e}}$ siècle à devenir les jardins familiaux pris dans les tensions foncières des banlieues résidentielles actuelles. Dans une seconde partie, il décrit le processus de diversification des formes de jardins collectifs qui a entraîné un fort développement des jardins partagés et du jardinage de rue. Il finit par s'interroger sur la portée patrimoniale des 12 sites de jardins familiaux et des 53 sites de jardins partagés de Marseille.

Based on an analysis of historical archives and long-term monitoring of the situation in the city, this article proposes a geo-historical interpretation of collective gardens in Marseille. It identifies the ideas that, at national and local levels, bring about the changes in these gardens, the stakeholders who are involved in them and the urban spaces that are designated for them. The first part of the article comprises a study of the territorial process whereby workers' allotments located in the agricultural suburbs of the early twentieth-century city tend to become family gardens subjected to all the real estate pressures of today's residential suburbs. In a second part, the article gives an account of the diversification of collective gardens with a notable development of community gardens and street gardening. Finally, it wonders what sort of heritage status might be attributed to the city's twelve family allotment gardens and fifty or so shared community gardens.

\section{INDEX}

Mots-clés : jardins collectifs, jardins familiaux, jardins partagés, géohistoire, Marseille

Keywords : collective gardens, allotment gardens, community garden, geo-history, Marseilles

\section{AUTEUR}

\section{JEAN NOËL CONSALÈS}

Maître de conférences en urbanisme, aménagement du territoire et géographie à l'Université d'Aix-Marseille jean-noel.consales@univ-amu.fr 